\title{
Simple Formulae for Heavy Ion Subcoulomb Transfer Probabilities. Application to One and Two-Proton Transfers in Bismuth
}

\author{
R. Bimbot and D. Gardès \\ Laboratoire de Chimie Nucléaire, Institut de Physique Nucléaire, Orsay, France \\ J. Cugnon ${ }^{\star}$ and R. da Silveira \\ Division de Physique Théorique ${ }^{\star \star}$, Institut de Physique Nucléaire, Orsay, France
}

Received November 20, 1978

\begin{abstract}
A simple analytical formula is given to express the transition probability in a quasi-elastic transfer reaction. This formula is derived from the DWBA amplitude, using assumptions based on the fact that the main contribution to the DWBA integral comes from a narrow domain of distances of approach. Recoil effects are taken into account. This method is applied to calculate the excitation functions for one and two-proton transfer reactions induced in ${ }^{209} \mathrm{Bi}$ by heavy ions, below and near the barrier. The curves obtained are compared to the excitation functions measured for the production of residual ${ }^{210} \mathrm{Po}$ and ${ }^{211}$ At. The corresponding transfer reactions involve excitation energies $E^{*}$ of the residue lying in the range $0-8 \mathrm{MeV}$. This excitation energy is taken as a free parameter in the calculation, and the values $E_{\mathrm{opt}}^{*}$ which lead to the best agreement between the calculated and experimental curves are consistent with the classical values for the optimum reaction $Q$ values.

Nuclear Reactions. Simplified transition probability for quasi-elastic transfer. Calculated $\sigma(E){ }^{209} \mathrm{Bi}(X, Y)^{210} \mathrm{Po},{ }^{211} \mathrm{At}, X={ }^{14} \mathrm{~N},{ }^{16} \mathrm{O},{ }^{19} \mathrm{~F},{ }^{40} \mathrm{Ar},{ }^{40} \mathrm{Ca},{ }^{56} \mathrm{Fe},{ }^{63} \mathrm{Cu}$ near threshold. Compared experimental data. Deduced optimum excitation energy.
\end{abstract}

\section{Introduction}

Since the early papers of Breit et al. [1,2], quasielastic particle transfer reactions induced by heavy ions have been extensively studied $[3,4]$. Several theoretical approaches of the problem have been described, the most fruitful ones using the distorted wave Born approximation (DWBA). This treatment, initially limited to neutron transfer, has been extended to charged particle transfer by Buttle and Goldfarb $[5,6]$, who also showed that recoil effects are important. However, this formalism is rather complicated and it is not always obvious how to extract, from these calculations, the salient physical features of the processes involved. In particular, the DWBA method is not able to express clearly the relative influence of some physical parameters, such as the incident energy, or the optimum $Q$-value, on the transfer probability. For example, in [7], the exci-

* Permanent address: Institut de Physique, Université de Liège, 4000 Sart Tilman, B-4000 Liege 1, Belgium

$\star \star$ Laboratoire associé au C.N.R.S. tation functions for transfer reactions could be qualitatively interpreted, below the barrier, by the combined effects of an energetic and a geometrical factor: the cross sections for a given reaction at sub barrier energies are significant if the energetic balance (potential energy + mass balance) is positive and if the distance of approach is small enough. Another simple model was proposed in Ref. [8] to explain the $Q$ value dependence of the cross sections for few nucleon transfers. According to this model, the distribution of final states in the residual nucleus corresponds to the maximal entropy, subject to a kinematical constraint on the excitation energy, in order to ensure a $Q$-value distribution centered around a given value. Such effects do not appear clearly from DWBA treatments. Moreover, despite significant improvements, the DWBA codes are still time consuming and are not adapted for the interpretation of low-resolution experiments in which the summation on many final states cannot be avoided. Finally, it has recently been 
shown that the DWBA formalism does not account for the energy dependence of the cross sections for transfer reactions to individual states $[9,10]$. The suggestion was made that this failure might be due to the neglect of competition with the large group of unresolved states which we consider here. Thus, on many counts, it is important to try to develop a description of transfer reactions to many unresolved states.

In this paper we propose a simplification for the expression of the DWBA amplitude based on the often quoted fact that, for subbarrier on nearbarrier quasi-elastic particle transfer, the main contribution to the DWBA integral comes from a narrow domain of the $r$-space, $r$ being the relative distance of the two fragment centers. This formulation, which is based on the principles given in [11], will allow us to get the transition probability in a closed analytical form. The same approach was used in [12] for neutron transfer reactions.

In Sect. 1, we describe the general method for deriving the transition probability and the basic assumptions. Then, explicit formulae are given in Sect. 2, and discussed in Sect. 3. In Sect. 4, the method is used to calculate the excitation functions for one and twoproton transfer reactions, and the results are compared to the experimental data of [13].

\section{The Method}

Let us consider a nuclear reaction in which a particle is transferred from a light projectile to a heavy target (process denoted $L p$ type transfer in [6]), for an incident energy lower than the interaction barrier. Such a reaction may be considered as a transition from an initial state 1 (projectile + target, with orbital angular momentum $l_{1}$ at a given distance $R_{1}$ ) to a final state 2 which is defined by the respective excitation energies, intrinsic and orbital angular momenta of the final nuclei, situated at a distance $R_{2} \simeq R_{1}$. For low incident energies, the transfer probabilities will be mostly influenced by the potential energies relative to the entrance and exit channels. A typical situation, which corresponds to head-on collisions, zero angular momentum transfer, and ground stateground state transitions, is shown in Fig. 1, where the potential energies in the entrance and exit channels are plotted versus the radial distance $r$, with the respective indexes 1 and 2 . In order to take into account the mass balance of the reaction $Q_{g g}$, defined as the difference between the sums of final and initial masses: $Q_{\mathrm{gg}}=\Sigma M_{2}-\Sigma M_{1}$, the quantities $U_{1}=V_{1}$ and $U_{2}=V_{2}-Q_{g g}$ are compared in Fig. 1. The interaction potentials $V_{1}$ and $V_{2}$ are calculated for the $s$ wave, and

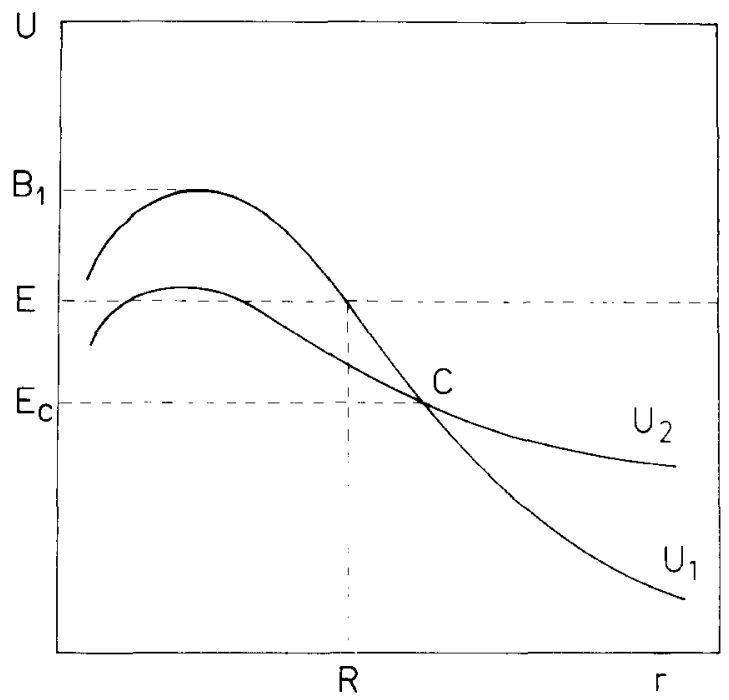

Fig. 1. Potential energies in the entrance and exit channels. The quantities $U_{1}=V_{1}$ and $U_{2}=V_{2}-Q_{g}$ are plotted versus the radial distance $r . V_{1}$ and $V_{2}$ denote the interaction potentials in the entrance and exit channels respectively, and $Q_{g g}$ the ground-state mass difference of the reaction. The crossing point $C$ has a crucial influence on the transfer probability. $B_{1}$ is the interaction barrier in the entrance channel

are the sum of the nuclear and Coulomb terms. From the comparison of such curves and from experimental data, it was inferred in [7] that the transfer cross sections become significant for energies higher than the crossing point energy $E_{c}$, provided that the value of $r$ is small enough to ensure a sufficient nuclear matter overlap.

If the reaction considered leads to a final state characterized by an excitation energy $E^{*}$, the quantity $U_{2}$ becomes equal to $V_{2}-Q$, with $Q=Q_{\mathrm{gg}}-E^{*}$. Moreover, if the initial and final angular momenta are different from zero, one should take into account a centrifugal term in the expression of the interaction potential (see below: Eq. (9)).

For the $L p$ transfers considered here, the $Q_{g g}$ values are always negative, and the transition amplitude will be calculated in the post interaction representation [6].

The transition probability $\omega$ from state 1 to state 2 is expressed in the frame of the Born approximation by:

$\omega=\frac{2 \pi}{\hbar}\left|\int_{0}^{x} \chi_{2}^{*}(r) \cdot F(r) \cdot \chi_{1}(r) d r\right|^{2}$

where $\chi_{j}$ is the wave function, suitably normalized, for the relative motion in channel $j(j=1,2)$ and $F(r)$ the form factor.

Our aim is to provide sufficiently accurate approximations which transform expression (1) into a much simpler one, that we hope to be analytical. In order 
to introduce these approximations, a few remarks must be made. As it has been extensively discussed (see Refs. $[6,14]$ ) the variation of the relative phase of the functions $\chi_{1}$ and $\chi_{2}$ is becoming very fast as $r$ is going away from the classical turning points. Hence an accurate description of the functions $\chi_{j}$ is needed only in the vicinity of these classical turning points, provided it respects the behaviour of the relative phase, at larger $r$. Such a description is realized by approximating the Coulomb potentials by constant gradient potentials in the region of interest, that is to say by replacing the potential energy curves by their tangents. The tangential point is chosen as the one corresponding to the outermost distance of closest approach. In order to respect the energy conservation laws, this distance is defined (see Fig. 1), as the interaction of the curve $U=E$ ( $E$ being the c.m. incident energy) with the more external potential energy curve encountered. This curve corresponds to the entrance or exit channels according to the relative position of the curves $U_{1}=f(r)$ and $U_{2}=f(r)$. The distance of closest approach corresponding to this tangential point is denoted by $R$.

As $r$ retreats from $R$, the product $\chi_{2}^{*} \chi_{1}$ rapidly vanishes, and the estimation of the form factor may be limited to a narrow domain around $R$. In this region, $F(r)$ can be approximated by the asymptotic form of an Hankel function [3-6, 14]. Its variation is not significant in the region of interest, and $F(r)$ has been approximated by $F(R)$. It can then be factored out of the integral. Moreover, since $\chi_{j}(r)$ decreases rapidly in the region $r<R$, the lower limit of the integral in (1) can be extended to $-\infty$. The expression of $\omega$ then becomes:

$$
\begin{aligned}
& \omega=\frac{2 \pi}{\hbar} F^{2}(R)\left|\int_{-\infty}^{\infty} \chi_{2}^{*}(r) \chi_{1}(r) d r\right|^{2} \\
& =\frac{2 \pi}{\hbar^{2}} F^{2}(R)\left|\int_{-\infty}^{+\infty} \gamma_{2}^{*}(p) \gamma_{1}(p) d p\right|^{2}
\end{aligned}
$$

where $\gamma_{1}$ and $\gamma_{2}$ are the Fourier transforms of $\chi_{1}$ and $\chi_{2}$ :

$\gamma_{j}(p)=\frac{1}{(2 \pi h)^{1 / 2}} \int e^{i / h p_{r}} \chi_{j}(r) d r \quad$ for $j=1,2$.

The calculation of the wave functions $\gamma_{j}(p)$ in a constant gradient potential is well known [15]. The resulting expressions are:

$$
\begin{aligned}
& \gamma_{1}(p)=\left|f_{1}\right|^{-1 / 2} \exp \left[\frac{i}{\hbar f_{1}}\left(\left(E-U_{1}\right) p-\frac{p^{3}}{6 \mu_{1}}\right)\right] \\
& \gamma_{2}(p)=\left|2 \pi \hbar f_{2}\right|^{-1 / 2} \exp \left[\frac{i}{\hbar f_{2}}\left(\left(E-U_{2}\right) p-\frac{p^{3}}{6 \mu_{2}}\right)\right]
\end{aligned}
$$

where $U_{1}$ and $U_{2}$ are the potential energy values for $r$ $=R$, and where $f_{1}$ and $f_{2}$ are the classical forces acting at this point:

$f_{j}=-\left.\frac{\partial U_{j}(r)}{\partial r}\right|_{r=R} \quad$ for $j=1,2$.

The terms $\mu_{j}$ are the reduced masses in channel $j$. Equations (2), (3), (4) yield

$$
\begin{aligned}
& \omega=\left.\frac{F^{2}(R)}{\hbar^{2} f_{1} f_{2}}\right|_{-\infty} ^{+\infty} \exp \frac{i}{\hbar}\left[\left[\left(\frac{1}{f_{1}}-\frac{1}{f_{2}}\right) E-\left(\frac{U_{1}}{f_{1}}-\frac{U_{2}}{f_{2}}\right)\right] p\right. \\
& \left.-\frac{p^{3}}{6}\left(\frac{1}{\mu_{1} f_{1}}-\frac{1}{\mu_{2} f_{2}}\right)\right]\left.d p\right|^{2} .
\end{aligned}
$$

Using the transformation

$\frac{p^{3}}{6 \hbar}\left(\frac{1}{\mu_{1} f_{1}}-\frac{1}{\mu_{2} f_{2}}\right)=-\frac{u^{3}}{3}$

and taking into account the definition of the Airy function

$A(X)=\frac{1}{\pi} \int_{0}^{x} \cos \left(u X+\frac{u^{3}}{3}\right) d u$.

Equation (5) can be rewritten:

$\omega=\frac{4 \pi^{2}}{\hbar^{2}} \frac{F^{2}(R)}{f_{1} f_{2}}\left(\frac{2 \hbar}{\frac{1}{\mu_{1} f_{1}}-\frac{1}{\mu_{2} f_{2}}}\right)^{2 \cdot 3} A^{2}(X)$

with

$X=-\frac{1}{\hbar}\left[\left(\frac{1}{f_{1}}-\frac{1}{f_{2}}\right) E-\left(\frac{U_{1}}{f_{1}}-\frac{U_{2}}{f_{2}}\right)\right]\left(\frac{2 \hbar}{\frac{1}{\mu_{1} f_{1}}-\frac{1}{\mu_{2} f_{2}}}\right)^{13}$

For incident energies $E$ lower than $E_{c}$, i.e. below the crossing point energy, the distance of closest approach is defined by $E=U_{2}$ (see Fig. 1), and the expression $X$ becomes:

$X=-\frac{E-V_{1}(R)}{f_{1}}\left(\frac{2}{h}\right)^{2 / 3}\left(\frac{1}{\mu_{1} f_{1}}-\frac{1}{\mu_{2} f_{2}}\right)^{-1 / 3}$.

On the other hand, for $E>E_{c}, X$ is given by

$X=\frac{E-V_{2}(R)+Q_{g g}}{f_{2}}\left(\frac{2}{h}\right)^{2 / 3}\left(\frac{1}{\mu_{1} f_{1}}-\frac{1}{\mu_{2} f_{2}}\right)^{-1 / 3}$.

\section{Explicit Formulae}

\subsection{Form Factor}

A suitable approximation for the form factor for subcoulomb energies is given by the expression [3, 
16]:

$F(r)=N \cdot \frac{e^{-\alpha r}}{\alpha r}$

where $\alpha$ is related to the binding energy $B$ of the transferred particle of mass $m_{t}$ in the final nucleus:

$\alpha=\left[2 m_{t} B / \hbar^{2}\right]^{1 / 2}$.

The term $N$ in Eq. (7) contains the spectroscopic factor $(S)$ and a normalization term. In the present case, the spectroscopic factors are not known, and $N$ has been arbitrarily taken to be equal to 1 . We thus obtain relative values for the cross sections, but the shapes of the calculated excitation functions will not be affected, as $N$ is independent of incident energy or angular momentum, for a given transfer reaction.

\subsection{Interaction Potential}

The total real potential is written as

$V_{j}(r)=V_{N_{j}}+\frac{Z_{1 j} Z_{2 j} e^{2}}{r}+\frac{\hbar^{2}}{2 \mu_{j}} \frac{l_{j}\left(l_{j}+1\right)}{r^{2}}$

where $V_{N j}, Z_{1 j}, Z_{2 j}, \mu_{j}$ are respectively the nuclear potential, the projectile and target charges, and the reduced masses, and $l_{j}$ the angular momentum for the channel $j(j=1,2)$. The real part of the nuclear potential is deduced from the energy density formalism (Ref. 17)

$V_{N}(r)=\frac{A_{1}^{1 / 3} A_{2}^{1 / 3}}{A_{1}^{1 / 3}+A_{2}^{1 / 3}} \cdot U_{N}(r)$

with

$U_{N}(r)=-V_{0} \exp \left(-0.27\left(r-r_{0}\left(A_{1}^{1 / 3}+A_{2}^{1 / 3}\right)\right)\right.$.

$V_{0}$ and $r_{0}$ being equal respectively to $-30 \mathrm{MeV}$ and $1 \mathrm{fm}$ for all the systems.

\subsection{Introduction of Recoil Effects}

It was underlined in [6] that taking into account the recoil effects, which arise from the modification of the center of mass coordinates when the transfer takes place, gives a great improvement in the general treatment of the transition amplitude.

Recoil corrections have been introduced here according to the method given by Buttle and Goldfarb [6]. The transferred particle being denoted $t$, and $c_{1}$ and $c_{2}$ denoting the nucleus cores, the transfer reaction is schematized as follows:

$$
\left(c_{1}+t\right)+c_{2} \rightarrow c_{1}+\left(c_{2}+t\right)
$$

Here, $a_{1}=\left(c_{1}+t\right)$ is the projectile, $a_{2}=\left(c_{2}+t\right)$ is the nucleus formed after the target $c_{2}$ has captured the particle $t$.

The wave functions $\chi_{1}$ and $\chi_{2}$ are now connected with the potential energy curves considered as functions of the relative distance $r$ of the cores. Near the furning points the relative distances $r_{1}$ and $r_{2}$ of the ions in the entrance and exit channels are related by:

$r_{1}=\xi_{1} r \quad r_{2}=\xi_{2} r$

where

$\xi_{1}=1-\frac{m_{t}}{m_{a_{1}}} \frac{R_{1}}{\bar{r}} \quad \xi_{2}=\frac{m_{c_{2}}}{m_{a_{2}}}+\frac{m_{t}}{m_{a_{2}}} \frac{R_{1}}{\bar{r}}$.

The masses $m_{a_{1}}, m_{a_{2}}, m_{c_{2}}$ and $m_{t}$ refer to the different nuclei and particles defined above, and $\bar{r}$ is the closest distance of approach, averaged for the entrance and exit channels.

The recoil correction leads us to replace in $(6 \mathrm{a}),(6 \mathrm{~b})$, (6c) the expressions of $U_{1}, U_{2}, f_{1}, f_{2}$ by their corrected evaluation: $U_{1}\left(\xi_{1} r\right), U_{2}\left(\xi_{2} r\right)$

$f_{1}=-\frac{\partial U_{1}\left(\xi_{1} r\right)}{\partial r} \quad f_{2}=-\frac{\partial U_{2}\left(\xi_{2} r\right)}{\partial r}$

and the method of Sect. 1 can be applied again. We give the final result which generalizes (6). If $E<E_{c}, r$ is solution of the equation $E=U_{2}\left(\xi_{2} r\right)$ and

$$
\begin{aligned}
& \omega=\frac{4 \pi^{2}}{\hbar^{2}} \frac{F^{2}(r)}{f_{1} f_{2}}\left(\frac{2 \hbar}{\frac{1}{\mu_{1} f_{1}}-\frac{1}{\mu_{2} f_{2}}}\right)^{2 / 3} \\
& \cdot A^{2}\left[-\frac{E-U_{1}\left(\xi_{1} r\right)}{f_{1}}\left(\frac{2}{\hbar^{2}}\right)^{1 / 3}\left(\frac{1}{\mu_{1} f_{1}}-\frac{1}{\mu_{2} f_{2}}\right)^{-1 / 3}\right]
\end{aligned}
$$

where $f_{1}$ and $f_{2}$ are given by (15).

If $E>E_{c}, r$ is solution of $E=U_{1}\left(\xi_{1} r\right)$ and

$$
\begin{aligned}
& \omega=\frac{4 \pi^{2}}{\hbar^{2}} \frac{F^{2}(r)}{f_{1} f_{2}}\left(\frac{2 \hbar}{\frac{1}{\mu_{1} f_{1}}-\frac{1}{\mu_{2} f_{2}}}\right)^{2 / 3} \\
& \cdot A^{2}\left[\frac{E-U_{2}\left(\xi_{2} r\right)}{f_{2}}\left(\frac{2}{\hbar^{2}}\right)^{1 / 3}\left(\frac{1}{\mu_{1} f_{1}}-\frac{1}{\mu_{2} f_{2}}\right)^{-1 / 3}\right] .
\end{aligned}
$$

\section{Discussion}

Equations (16) show that the transition probability is dominated by two factors:

(i) $F(r)$ which expresses the effect of the range of the nuclear force responsible for the transfer 


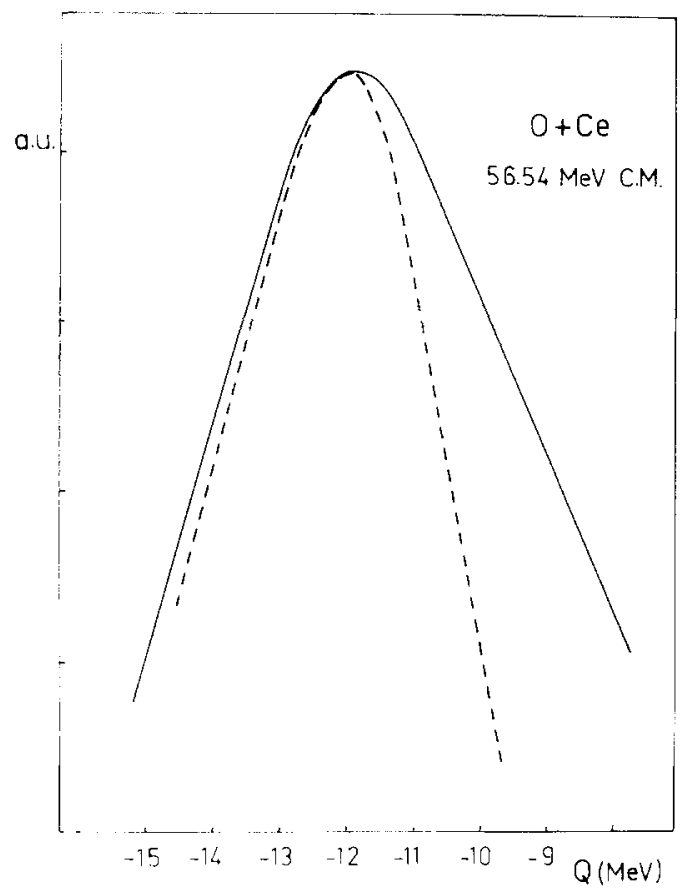

Fig. 2. Variation of the transfer probability versus the $Q$-value. The dotted line represents the transfer probability calculated for $l=0$ using the present method, the solid line a DWBA calculation of the differential cross section for $\theta_{L}=150^{\circ}$, taken from [13]. The transfer reaction considered is ${ }^{16} \mathrm{O}+{ }^{140} \mathrm{Ce} \rightarrow{ }^{14} \mathrm{C}+{ }^{142} \mathrm{Nd}$, and the incident energy is $56.5 \mathrm{MeV}$ in the c.m.s. The curves have been normalized at their maximum

(ii) the Airy function which shows that the transfer yield is considerably influenced by the matching of the relative motion wave functions. This factor characterizes the energetics of the reaction: for $L p$ type transfers, the transfer yield is dramatically reduced for energies lower than $E_{c}$, because the argument of the Airy function is positive for $E<E_{c}$, and the function decreases exponentially.

On the other hand, when $E>E_{c}$, the argument becomes negative and the function goes through a maximum. By taking into account the properties of the Airy function, Eq. ( $6 \mathrm{c})$ has been used to derive an analytical expression for the optimum $Q$-value $\left(Q_{\text {opt }}\right)$ in a simple case (no angular momentum transfer $(\Delta l=0)$, zero initial angular momentum $(l=0))$. This expression is given in [11].

The dependence of the transfer probability on the $Q$ value has been calculated under these assumptions $l$ $=0, \Delta l=0$, and compared to the result of a full DWBA calculation. The chosen reaction was the two-proton transfer reaction ${ }^{16} \mathrm{O}+{ }^{140} \mathrm{Ce} \rightarrow{ }^{14} \mathrm{C}$ $+{ }^{142} \mathrm{Nd}$ which has been studied previously by Von Oertzen et al. [18]. The comparison is shown in Fig. 2. It can be seen that a fair agreement is obtained, particularly for the position of the maximum, which defines the optimum $Q$-value. This indicates that the approximations involved in the present work lead to results which are accurate enough, at least as far as the dependence of the transfer probability on the $Q$-value is concerned (or its dependence on the incident energy, since $E$ and $Q$ play symmetric roles in the formalism).

\section{Application to Excitation Functions}

For a given transfer reaction, at a given energy, Eqs. (16a) and (16b) can be used to calculate the transfer probability $\omega\left(l_{1}\right)$ for each partial wave in the entrance channel. It is then possible to get the transfer cross section by multiplying $\omega\left(l_{1}\right)$ by the cross section for elastic scattering corresponding to the same angular momentum. Below the interaction barrier, this Rutherford cross section can be approximated by the term

$\sigma\left(l_{1}\right)=\left(2 l_{1}+1\right) \pi \lambda^{2}$

where $\hat{\lambda}$ is the reduced de Broglie wave length for the relative motion.

The total cross section for the transfer reaction considered, at the energy $E$, is then given by the summation

$\sigma(E)=\pi \lambda^{2} \sum_{l_{1}=0}^{l_{\max }}\left(2 l_{1}+1\right) \omega\left(l_{1}\right)$.

Practically, the value $l_{\max }$ has been chosen to be the lowest $l$ value which gives a contribution smaller than $0.1 \%$ to the sum.

This method has been used to calculate excitation functions for one and two-proton transfer reactions, induced with heavy ions in Bi targets, close to their energy threshold. This was done in order to compare the calculated values with the experimental data from [13]. In this work, one and two-proton transfers induced in $\mathrm{Bi}$ were studied by observation of the heavy residual nuclei ${ }^{210} \mathrm{Po}$ and ${ }^{211} \mathrm{At}$. The identification of the residual nucleus is generally not sufficient to determine unambiguously the nuclear reaction which has taken place. For example, ${ }^{211} \mathrm{At}$ can be produced either through direct transfer of two protons from projectile to target, or through the transfer of two protons and $x$ neutrons, followed by $x$ neutron evaporation from the excited ${ }^{211+x} \mathrm{At}$ nucleus. But these various reaction channels correspond to different energetics and can be distinguished from each other on the basis of the c.m. energy distribution of the residual nuclei. This was done for the lightest projectiles used $\left({ }^{14} \mathrm{~N},{ }^{16} \mathrm{O}\right)$ [19]. For incident energies close to the thresholds, the reactions leading to ${ }^{210} \mathrm{Po}$ and ${ }^{211} \mathrm{At}$ involve the transfer from projectile 

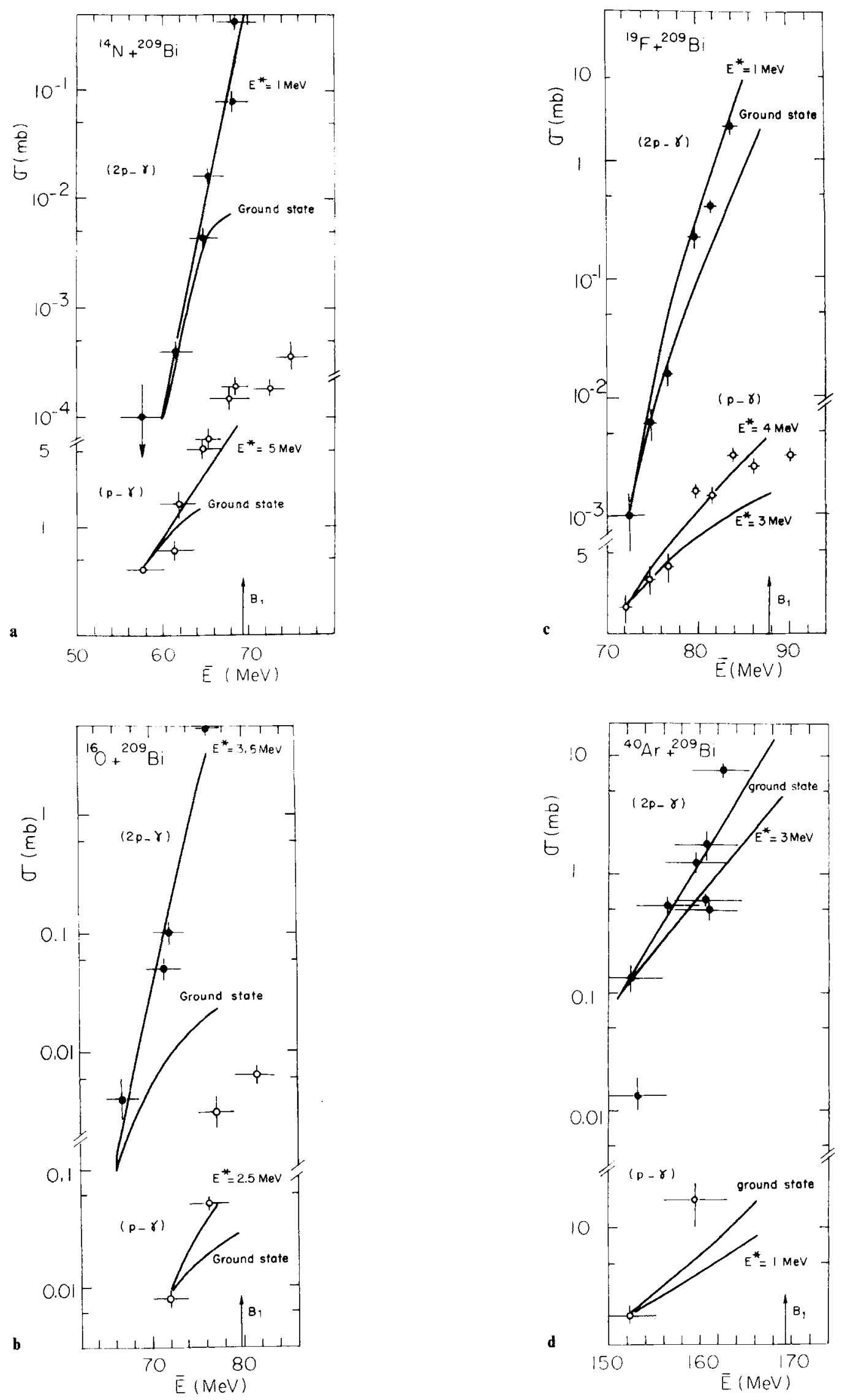

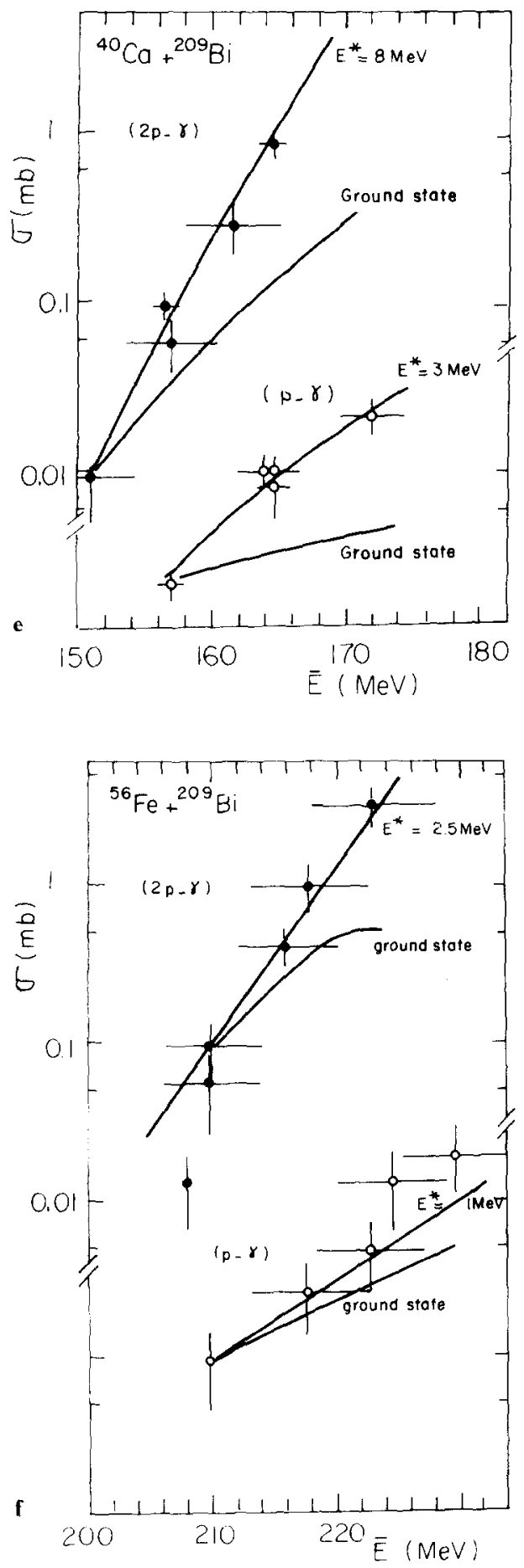

to target, of one and two protons respectively, the ${ }^{210}$ Po or ${ }^{211}$ At nuclei being formed with an excitation energy lower than the neutron separation energy $B_{n} \simeq 8 \mathrm{MeV}$. Such reaction paths are denoted $(+p,-\gamma)$ and $(+2 p,-\gamma)$. Under the assumption that this result can be extended to the other projectiles, all the physical parameters which enter the calculation (incident energy, masses and atomic numbers in the entrance and exit channels, $Q_{\mathrm{gg}}$ ) are defined, with the

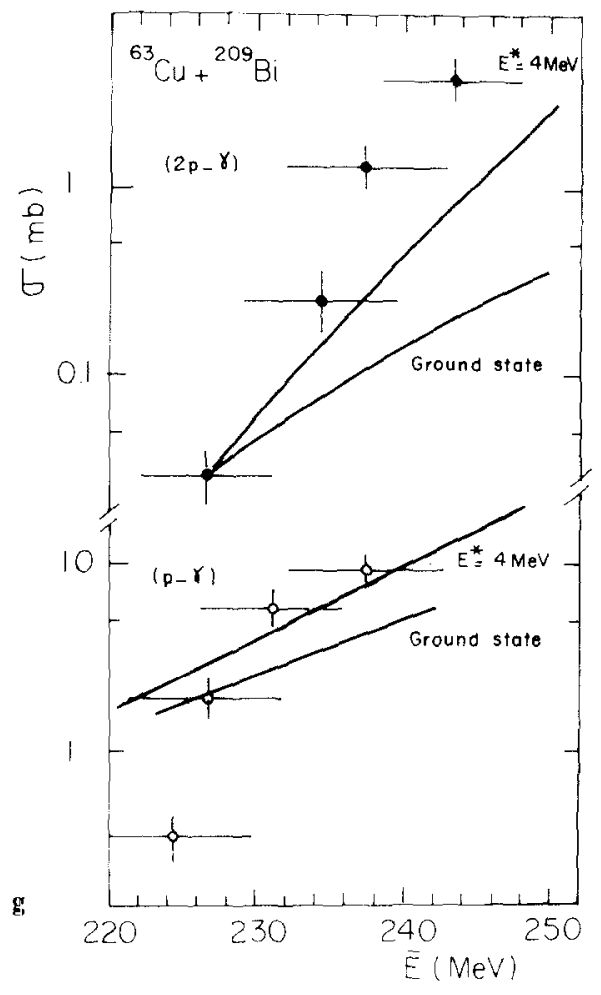

Fig. 3a g. Comparison of the calculated excitation functions (solid lines) to the experimental cross sections (points associated with error bars). The black symbols refer to 2 proton transfers, the open ones to one proton transfer. The system projectile + target is indicated in each case. The excitation energy $E^{*}$ adopted for each calculation is indicated close to the curve. The calculated excitation functions have been normalized to one experimental point. The vertical arrow indicates the position of the calculated interaction barrier $B_{1}$

exception of the excitation energy $E^{*}$ and orbital angular momentum $l_{2}$ of the system in the exit channel. Assuming that most of the excitation energy is concentrated on the heavy reaction product, the parameter $E^{*}$ has been considered as free within the above mentioned limits of 0 and $8 \mathrm{MeV}$. Moreover, the angular momentum transfer has been assumed to be negligible so that $l_{2}=l_{1}$.

The result of this calculation, for one and two- 
proton transfer reactions induced in ${ }^{209} \mathrm{Bi}$ by ${ }^{14} \mathrm{~N}$, ${ }^{16} \mathrm{O},{ }^{19} \mathrm{~F},{ }^{40} \mathrm{Ar},{ }^{40} \mathrm{Ca},{ }^{56} \mathrm{Fe}$ and ${ }^{63} \mathrm{Cu}$ are compared in Fig. 3 to the experimental excitation functions from [13], in the subcoulomb energy range. The points associated with error bars represent the experimental cross sections, and the solid line the calculated excitation functions, after arbitrary normalization. As mentioned previously, recoil effects have been taken into account in the calculation, and the potential energy functions include a nuclear and centrifugal part.

The parameter $E^{*}$ has a significant influence on the slope of the excitation function. This can be appreciated by comparing the curves obtained for $E^{*}=0$ (ground state to ground state transitions) to those which lead to the best fit, for which the value of $E^{*}$ (denoted $E_{\mathrm{opp}}^{*}$ ) is indicated in the figures. It can be seen that very good agreement between experiment and calculation is obtained for all projectiles, and that the corresponding value of $E_{\mathrm{opt}}^{*}$ always lies in the range $0-8 \mathrm{MeV}$ previously defined from the experimental studies.

It is obvious that the excitation functions obtained in [13] do not correspond to a single energy level in the exit channel. Therefore, the value of $E_{\mathrm{opt}}^{*}$ must be considered as the mean energy of the group of levels towards which the transfer occurs preferentially. It is therefore interesting to compare the values $E_{\mathrm{opt}}^{*}$ found from the present calculation to the optimal excitation energies which can be derived

i) from the classical expression of the optimal $Q$ value:

$Q_{\mathrm{opt}}=E\left(\frac{Z a_{1} Z c_{2}}{Z a_{2} Z c_{1}}-1\right)$

and the corresponding excitation energy

$E_{0}^{*}=Q_{\mathrm{gg}}-Q_{\mathrm{opt}}$

ii) from the expression $Q_{g g}-\Delta V$ which was shown in [7] to be equivalent to the optimum excitation energy, but which takes into account the nuclear part of the interaction potential. The quantity $\Delta V=V_{2}(R)$ $-V_{1}(R)$ is calculated for the value $R$ of the radial distance such that $E=V_{1}(R)$.

This comparison is made in Table 1 . For each of expressions (i) and (ii), two values are given which correspond to the limits of the energy range over which the experimental and theoretical curves are compared, the upper limits being equal to the interaction barrier. Although negative values for the excitation energies have no physical meaning, they have been kept in Table 1, in order to appreciate the amount of energy missing to reach the ground state level $\left(E^{*}=0\right)$. First it can be seen from Table 1 that
Table 1. Comparison of the optimum excitation energies $E_{0 \mathrm{ot}}^{*}$ leading to the best fit of experimental data with the classical values

i) $E_{0}^{*}=Q_{\mathrm{gg}}-Q_{\mathrm{opt}}$

ii) $Q_{g g}-\Delta V$.

The expressions i) and ii) are calculated for two incident energies, the lowest energy of each excitation function $(L)$ and that of the calculated interaction barrier $(B)$

\begin{tabular}{lllllll}
\hline $\begin{array}{l}\text { Incident } \\
\text { ion }\end{array}$ & $\begin{array}{l}\text { Transfer } \\
\text { reaction }\end{array}$ & $\begin{array}{l}E_{\mathrm{opt}}^{*} \\
(\mathrm{MeV})\end{array}$ & $\begin{array}{l}Q_{g g}-\Delta V \\
(L)\end{array}$ & $\begin{array}{l}Q_{g g}-\Delta V \\
(B)\end{array}$ & $\begin{array}{c}E_{0}^{*} \\
(L)\end{array}$ & $\begin{array}{l}E_{0}^{*} \\
(B)\end{array}$ \\
\hline $\mathrm{N}$ & $+p,-\gamma$ & 5 & 4.5 & 6.7 & 5.0 & 6.6 \\
& $+2 p,-\gamma$ & 1 & -1 & 1.6 & -1.8 & 1.4 \\
$\mathrm{O}$ & $+p,-\gamma$ & 2.5 & 0.9 & 1.9 & -0.3 & 1.9 \\
& $+2 p,-\gamma$ & 3.5 & 0.9 & 4.1 & -0.5 & 3.9 \\
$\mathrm{~F}$ & $+p,-\gamma$ & 4 & 4.2 & 6.0 & 4.2 & 5.8 \\
& $+2 p,-\gamma$ & 1 & -1.4 & 2.2 & -1.3 & 2.0 \\
$\mathrm{Ar}$ & $+p,-\gamma$ & 0 & -0.6 & 0.1 & 0.9 & 0 \\
& $+2 p,-\gamma$ & 0 & 0.3 & 0.7 & -1.2 & 0.4 \\
$\mathrm{Ca}$ & $+p,-\gamma$ & 3 & 2.8 & 4.0 & 2.4 & 4.0 \\
& $+2 p,-\gamma$ & 8 & 4.8 & 8.5 & 5.0 & 8.2 \\
$\mathrm{Fe}$ & $+p,-\gamma$ & 1 & 0.3 & 1.5 & 0.4 & 1.3 \\
& $+2 p,-\gamma$ & 2.5 & 1 & 2.2 & 1 & 2.9 \\
$\mathrm{Cu}$ & $+p,-\gamma$ & 4 & 3.9 & 5.1 & 3.9 & 4.4 \\
& $+2 p,-\gamma$ & & 1.7 & 3.6 & 1.3 & 3.2 \\
\hline
\end{tabular}

the limits for the optimal excitation energy values calculated from both methods i) and ii) are very close to each other. This is not surprising because, for subcoulomb energies, the contribution of the nuclear potential can be neglected. Secondly, the value $E_{\mathrm{opt}}^{*}$ which best reproduces the slope of the experimental excitation function is always situated between the two limits of excitation energy defined above. This gives credence to the validity of the reaction paths $(+p,-\gamma)$ and $(+2 p,-\gamma)$ which have been assumed for the calculations, and to the physical meaning of the parameter $E^{*}$. The best agreement between the experimental and calculated curves is obtained when the exit channel corresponds to an energy level such that the potential energy curves $U_{1}$ and $U_{2}$ cross in an energy region close to the barrier. This result is in good agreement with the conclusions of [7], and the excellent fit obtained for two types of transfer reactions over a wide range of projectiles gives confidence in the validity of the approximations made for the transfer probability calculation.

\section{Conclusion}

We have worked out a simple analytical form for the transition probability for particle transfer reactions induced by heavy ions below the Coulomb barrier. It is based on the DWBA formalism and a reasonable 
approximation for the entrance and exit channels wave functions. The application of this formula to the calculation of cross sections leads to very good agreement with the excitation functions measured for the production of the residual nuclei, ${ }^{210} \mathrm{Po}$ and ${ }^{211} \mathrm{At}$ through one and two-proton quasi-elastic transfers. A single parameter $E_{\text {opt }}^{*}$ is used in the calculation, and the value of this parameter which gives the best fit

i) lies in the correct range $0-8 \mathrm{MeV}$ consistent with the observation of the residual nuclei

ii) is in good agreement with the value calculated from the classical expressions of the optimum $Q$ value.

This method of calculation is therefore very convenient for the interpretation of such data from low resolution experiments. Moreover, it has the advantage of leading to a factorized expression in which the relative influence of the energetic balance and of the distance of approach are more transparent.

We thank J.M. Alexander for interesting discussions and careful reading of the manuscript. One of us (J.C) is grateful for the hospitality extended to him by the members of the Division de Physique Théorique of the Institut de Physique Nucléaire at Orsay where this work has been performed.

\section{References}

1. Breit, G.. Hull, M.H., Jr., Gluckstern, R.L.: Phys. Rev. 87. 74 (1952)

2. Breit, G., Ebel, M.E.: Phys, Rev. 103, 679 (1956); Phys. Rev. 104, 1030 (1956)

3. Fleury, A., Alexander, J.M.: Ann. Rev. Nucl. Sci. 24, 279 (1974)

4. Von Oertzen. W.: Nuclear Spectroscopy. Cerny. J. (ed.). New York Acad. (1972)

5. Buttle, P.J.A., Goldfarb, L.J.B.: Nucl. Phys. A 115, 461 (1968)

6. Buttle, P.J.A., Goldfarb, L.J.B.: Nucl. Phys, A 176, 299 (1971)
7. Gardès, D., Bimbot, R., Fleury. A., Hubert, F., Rivet, M.F.: J. Phys. Lett. 36, L59 (1975)

8. Levine, R.D., Steadman, S.G.. Karp, J.S., Alhassid, Y.: Phys. Rev. Lett. 41, 1537 (1978)

9. Pieper, S.C. Macfarlane, M.H., Gloeckner, D.H., Kovar, D.G., Becchetti. F.D.. Harvey, B.G., Hendrie, D.L. Homeyer, H., Mahoney, J., Pühlhoffer, F., Von Oertzen. W.. Zisman. M.S.: Phys. Rev. C 18, 180 (1978)

10. Olmer, C.. Mermaz, M., Buenerd, M., Gelbke, C.K., Hendrie. D.L., Mahoney, J.. Scott. D.K., Macfarlane, M.H., Pieper, S.C.: Phys. Rev. C 18. 205 (1978)

11. Cugnon. J., Da Silveira, R., Gardès. D.: Proceedings Conf. Europ. Phys. Nucl avec des ions lourds. Caen 28 (1976). [PNO/TH 76-47

12. Cugnon, J.: Lettere al nuovo cimento, Vol. 19. N. 10, 361 (1977)

13. Gardès, D., Bimbot, R., Maison. J., de Reilhac. L., Rivet, M.F.. Fleury, A., Hubert, F., Llabador, Y.: Phys, Rev. C 18, 1298 (1978)

14. Nair. K.G., Blair. J.S., Reisdorf, W., Wharton, W.R., Braithwaite, W.J., Mehta. M.K.: Phys. Rev. C 8. 1129 (1973)

15. Landau, L.D., Lifchitz. E.M.: Quantum Mechanics. Oxford: Pergamon Press 1969

16. Broglia. R.A.. Winther, A.: Phys. Reports 4. 153 (1972)

17. Ngô, C.. Tamain, B.. Beiner, M., Lombard, R.J., Mas. D., Deubler, H.H.: Nucl. Phys. A 252, 237 (1975)

18. Von Oertzen. W.. Bohlen. H.G.. Gebauer, B.: Nucl. Phys. A 207, 91 (1973)

19. Gardès. D.: Thèse (Orsay) 1976

R. Bimbot

D. Gardès

Laboratoire de Chimie Nucléaire

Institut de Physique Nucléaire

$\mathrm{BP} \mathrm{n}^{\circ} 1$

F-91406 Orsay

France

J. Cugnon

R. da Silveira

Division de Physique Théorique

Institut de Physique Nucléaire

$B P n^{\circ} 1$

F-91406 Orsay

France 